and of the order associated with denaturation of proteins. Moreover the rate varies in first-order fashion with the concentration of the complex, and is independent of that of the free fragment, which indicates that the process is limited, as one would suppose, by the unfolding, or breathing frequency, rather than the reassociation rate, which is known from earlier studies to be fast. Now although the stability of the complex is somewhat lower than that of the integral enzyme, as judged by the thermal denaturation temperature, it may reasonably be inferred that the breathing equilibrium obtains in the latter. The cooperativity of the conformational equilibrium ensures that the native state will normally be overwhelmingly populated. Addition of the inhibitor, TDP and calcium ions, causes a dramatic reduction in the exchange rate by stabilizing the native structure.

The dynamic state of the native conformation, and the transient appearance of an unfolded form, is equally reflected in the kinetics of hydrogen exchange, an approach conceived long ago by Linderstrøm-Lang. The internal exchangeable hydrogens in a globular protein evidently become available by this mechanism, and Takesada, Nakanishi and Tsuboi (J. mol. Biol., 77, 605; 1973) have now extended their earlier analysis, based on the use of infrared spectroscopy to follow the exchange of peptide hydrogens against deuterium, to another protein, $\alpha$ lactalbumin. The supposition is that the unperturbed exchange rate for accessible protons is attenuated by a factor representing the equilibrium concentration of the unfolded form. Now the thermal denaturation profile can be used to yield the enthalpy and entropy of unfolding, and thence to derive the conformational equilibrium constant at temperatures outside the transition range, where the denatured state cannot be detected by spectroscopic methods. For lysozyme this constant leads to an excellent quantitative fit of the hydrogen exchange kinetics. There was also, as in staphylococcal nuclease, a large stabilization of the native state when specific inhibitors were introduced. In $\alpha$ lactalbumin there is a difference between the equilibrium constant for unfolding within the optical transition, and that inferred from hydrogen exchange at lower temperatures, which is most easily explained in terms of the existence of locally unfolded intermediates which govern the exchange rate. The number of slowly exchanging peptide hydrogens is thirtyfive in lactalbumin, compared with fortyfour in lysozyme, and this difference can be rationalized in terms of differences in the number of inferred intrapeptide hydrogen bonds in the conjectural lactalbumin structure, derived by fitting the sequence to the $\mathrm{X}$-ray model of 1ysozyme, to which it is closely related.

Local structural fluctuations also explain new tritium-exchange results of remarkable refinement, obtained by Englander and Rolfe (J. biol. Chem., 248,4852 ; 1973) on liganded and unliganded haemoglobin. This work represents the next stage of evolution of the method, for Englander has found that any transfer of exchangeable protons from a slower to a faster exchanging category, in consequence of a conformational perturbation of the protein, can be observed with great precision after partial tritiation of the protein in one of its two states, by measuring the exchange-out rates, first when it is in the same state, and then when it is in the other. By manipulating these combinations of exchange conditions, it is possible to obtain difference curves for exchange in the two states. Moreover, by choosing the time range (just as in relaxation experiments), from seconds to days, one can home in at will on the group of protons that show the most interesting differences. In haemoglobin, Englander and Rolfe find that there are three groups of protons, three, eight and nine in number, the exchange of which is ligand-sensitive. All of them exchange much faster in the liganded protein. Inasmuch as the rates are, as before, limited by the equilibrium between folded and (in this case locally) unfolded chain segments, an equilibrium constant and free energy can be attached to the conformational element corresponding to each group. A small energetic change, involving perhaps 2 $\mathrm{kcal} / \mathrm{mole}$, is sufficient to give rise to a readily measurable effect on the exchange, such as here observed. The greater local stability of the deoxyhaemoglobin befits what is regarded as the "relaxed" state, the haem ligand perturbing the conformation in the direc-

\title{
Immunoglobulin mRNa Sequences
}

THE exploratory work on messenger RNA sequences was for practical reasons confined to viral RNAs of Escherichia coli. The consequent development of highly sophisticated sequencing methods evolved to cope with the problems which arose in this work has opened up the possibility in future of sequencing other nucleic acids. The next obvious stage is to compare the sequences of these viral messengers (which are likely to possess distinctive functional sequences, for example, for replicative purposes) with those of normal cellular messengers, and to compare the sequences of eukaryotic and prokaryotic mRNAs, of both cellular and viral origin. Brownlee et al. (see Nature New Biology next Wednesday) have made an important step in this direction by determining the sequences of five long stretches within the mRNA chains for mouse immunoglobulin light chains from a myeloma tumour cell line grown in tissue culture.

The advantage of this system over many others from eukaryotes is the possibility of obtaining pure mRNA sufficiently highly labelled with ${ }^{32} \mathrm{P}$ for sequencing operations. Despite lack of mRNA contamination of the relevant 12-14S RNA fraction isolated by sucrose gradient centrifugation, there proved to be contamination with mitochondrial ribosomal RNA in the same size range. Brownlee et al. overcame this difficulty by using oligo(dT)cellulose column chromatography, the messenger being more tightly bound by virtue of its poly (A) "tail". Four out of five long sequences (more than eighteen residues) in the purified messenger were found to correspond, with reference to the genetic code, to known amino acid sequences in the corresponding mouse immunoglobulin light chains. Moreover, the authors were able to deduce from their results the actual triplet codons (where several might be possible owing to the degeneracy of the genetic code) which have been selected for specific amino acids in the immunoglobulin sequence. The nucleotide sequence of a fifth long fragment of the messenger does not correspond to any known amino acid sequence in the light chains and therefore is presumed to be located outside the coding region of the RNA.

The molecular weights of the immunoglobulin light chain mRNA and its 3'-poly (A) "tail", isolated after digestion of the intact mRNA with nucleases, were determined from the electrophoretic mobilities of these species in polyacrylamide gels containing formamide. It is concluded that the total length of the molecule is 1,200 residues and that of the poly (A) "tail" is 200 residues. By comparison, the poly (A) "tails" of HeLa cell mRNA are also about 200 residues long, but those of the mouse globin mRNAs are only $40-80$, and that of polio viral RNA is only about 90 ; histone mRNAs contain none. The coding region must be 690 residues long ( 3 times the number of amino acids). Subtracting this number plus 200 residues of the poly (A) "tail" from the total length of the molecule leaves 300 extra bases. It is not yet known how these are distributed between the $3^{\prime}$ and $5^{\prime}$ ends of the mRNA, but it is interesting to note that the $5^{\prime}$ terminus of the sequenced viral RNAs (MS2 and R17) and rabbit globin mRNA contains only about 150 extra bases at the $5^{\prime}$ end. 